\title{
Lipofuscin and melanin content of the retinal pigment epithelium in a case of Sjögren-Larsson syndrome
}

\author{
SVEN ERIK G NILSSON AND STEN JAGELL \\ From the Department of Ophthalmology, University of Linköping, and the Department of Paediatrics, Gävle \\ Hospital, Sweden
}

SUMmARY Necropsy material from the eye of a 23-year-old male known to have suffered from the Sjögren-Larsson syndrome, characterised by mental retardation, spastic diplegia or tetraplegia, congenital ichthyosis, and so-called glistening dots in the foveal and parafoveal areas of the fundus of the eye, was investigated ultrastructurally. The retinal pigment epithelium in the macular area showed a significant increase in lipofuscin granules, most likely because of a reduced capacity for digestion of phagocytosed material, and a significant decrease in melanin and melanolipofuscin granules in comparison with a normal control material. The ophthalmoscopically visible glistening dots and the pigment epithelial 'windows' seen on fluorescein angiography may possibly be explained by a further progression of these changes. No retinal changes were found in the macular area, the midperiphery, or the periphery that could be attributed with certainty to any other origin than post-mortem autolysis. More material would be needed, however, to elucidate further the fundal changes in the Sjögren-Larsson syndrome.

The Sjögren-Larsson syndrome, which is inherited in an autosomal recessive mode, is characterised by mental retardation, spastic diplegia or tetraplegia, and congenital ichthyosis. ${ }^{1}$ In addition specific ocular fundal changes are found, ${ }^{1}$ later described as 'glistening dots,' located in the foveal and parafoveal areas surrounding the foveola. ${ }^{24} \mathrm{Jagell}$ et al. ${ }^{4}$ found such slightly elevated glistening dots in all 30 patients who were subjected to fundal examination.

In fluorescein angiograms of their case Gilbert et $a l .^{2}$ observed increased transmission of the choroidal vascular pattern in scattered flecks within the macula, possibly indicating pigment epithelial 'windows.' In the same case the electroretinogram $a$ and $b$ waves were normal. ${ }^{2}$ The electro-oculogram (EOG) was normal in two patients subjected to this investigation. ${ }^{4}$ Conjunctival and corneal changes are considered to be part of the ectodermal disease. It has been suspected that the Sjögren-Larsson syndrome is associated with faulty metabolism of essential fatty acids. ${ }^{5}$

Correspondence to Dr S E G Nilsson, Department of Ophthalmology, University of Linköping, S-581 85 Linköping, Sweden.
The present paper reports on ultrastructural findings in the retina and pigment epithelium of an eye obtained as necropsy material from a subject with the Sjögren-Larsson syndrome.

\section{Material and methods}

The eye was enucleated at necropsy of a 23-year-old male known to have suffered from the SjögrenLarsson syndrome. After removal of the cornea the eye was immersed in $2.5 \%$ buffered glutaraldehyde solution about $10-11$ hours post mortem. After one week's fixation, the eye was cut into smaller pieces and postfixed in $1 \%$ osmium tetroxide in Veronal acetate buffer. Pieces of retina from the posterior pole, the midperiphery, and the periphery were dehydrated and embedded in Vestopal W. Thin sections were stained with uranyl acetate and lead citrate and examined in a Philips EM 300 electron microscope. Because of pronounced retinal detachment in the midperiphery and periphery, the pigment epithelium was studied only in the posterior pole.

For analysis of the content of lipofuscin, melanin, and melanolipofuscin granules, sections of pigment 


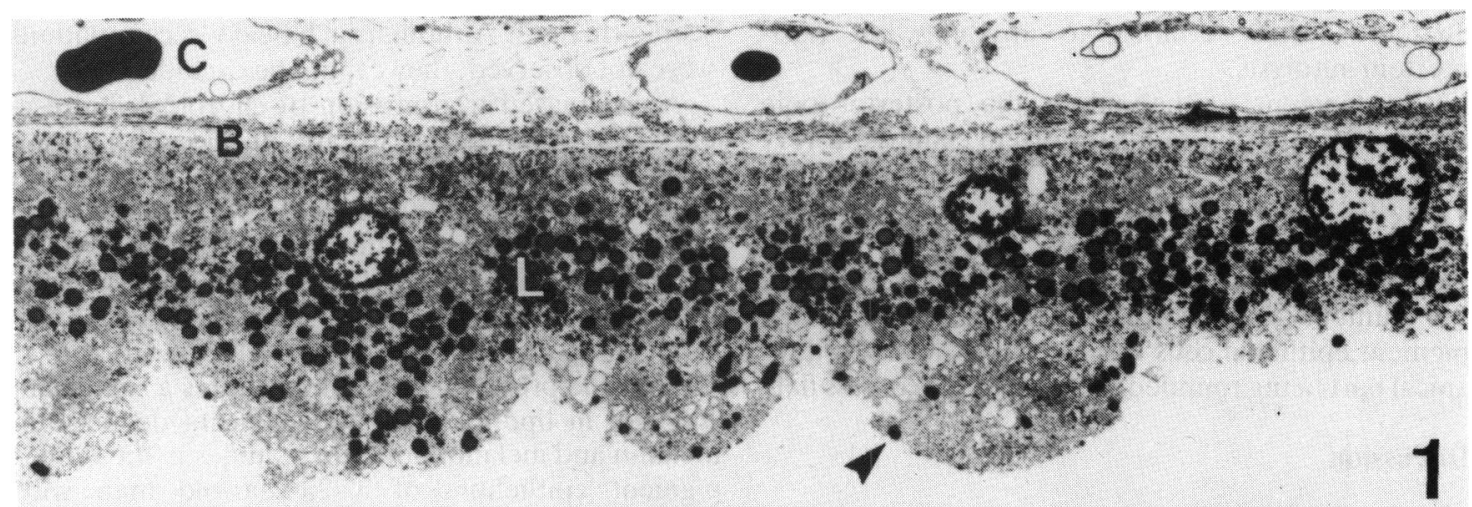

Fig. 1 Retinal pigment epithelium from a 23-year-old male with Sjögren-Larsson syndrome. Post-mortem material. A large number of lipofuscin granules (L) and fairly few melanin granules (arrowhead) are seen. B: Bruch's membrane. C: choroidal capillary.

epithelial cells were photographed at 3000 times magnification. The micrographs were printed at $\times 2 \cdot 5$. Montages of 15 contiguous pigment epithelial cell profiles were made from five different locations within the macular area.

\section{Results}

In spite of fairly extensive sectioning within the posterior pole, the midperiphery, and the periphery we did not find any retinal changes that could be

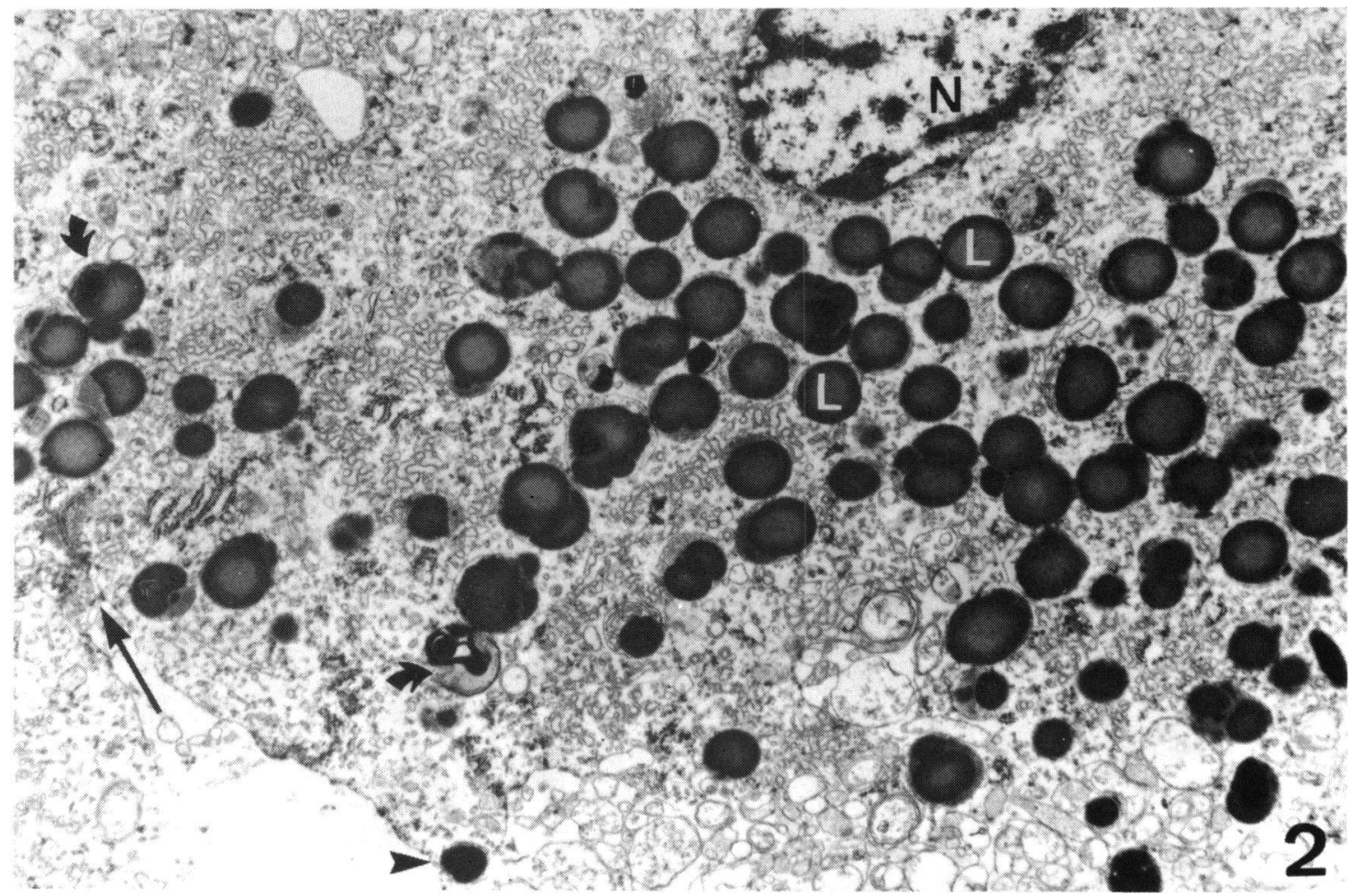

Fig. 2 Parts of two adjacent pigment epithelial cells from the same subject as in Fig. 1. Cell border at apical side indicated by long arrow. Abundant lipofuscin (moderately stained) granules (L), but much fewer melanin (totally black) granules (arrowhead) and melanolipofuscin (combinations of the two other types) granules (short arrow). N: nucleus. 
attributed with certainty to anything but postmortem autolysis.

The pigment epithelium of the posterior pole showed, in addition to post-mortem changes, a very high content of lipofuscin but a fairly low content of melanin (Figs. 1 and 2). The number of lipofuscin granules per cell profile was $55 \cdot 6 \pm 2.4$ (SEM), the number of melanolipofuscin granules $8 \cdot 5 \pm 0.5$, and the number of melanin granules $6 \cdot 8 \pm 0 \cdot 4$. Many pigment epithelial cells were very voluminous, their apical part being rounded instead of more or less flat.

\section{Discussion}

Feeney-Burns et al. ${ }^{6}$ performed careful morphometric analysis of the granular content of human pigment epithelial cells of eyes from each 10 decades of life. In the third decade, corresponding to the age of the present subject, the number per macular pigment epithelial cell profile of lipofuscin granules was about 15, the number of melanolipofuscin granules about 11, and the number of melanin granules $14.97 \pm 0.99$ (SEM). The number of lipofuscin granules was significantly $(p<0.001)$ higher (3-4 times), the number of melanolipofuscin ranules significantly $(p<0.05)$ lower, and the number of melanin granules significantly $(p<0.001)$ lower in the subject with Sjögren-Larsson syndrome than in the control material provided by FeeneyBurns et al. ${ }^{6}$

The reason for the prominent increase in lipofuscin content may be a decrease in pigment epithelial capacity for digestion of lipofuscin (phagosomes), or an increase in phagocytosed material, or both. However, apart from post-mortem changes the photoreceptor outer segments did not seem to show any signs of degeneration, a fact that speaks against an increase in phagocytosis.

The histopathology of the glistening dots is unknown. The cause may be inclusions in the retina. No retinal inclusions have been found, but their presence cannot be excluded either. It is also possible, however, that a very heavy accumulation of lipofuscin may form the basis of these ophthalmo- scopic changes. Although such heavy accumulations were not observed, they cannot be excluded.

An increased transmission of choroidal fluorescence was reported in a case of Sjögren-Larsson syndrome. ${ }^{2}$ The low content of pigment epithelial melanin may contribute to such an effect, but a true 'window' would most likely require even fewer melanin and melanolipofuscin granules or a pronounced pigment epithelial atrophy. No such areas were found, but they cannot be excluded.

Thus the present paper demonstrates a significant increase in lipofuscin and a significant decrease in melanin and melanolipofuscin granules in the retinal pigment epithelium of a 23-year-old man with Sjögren-Larsson syndrome in comparison with normal conditions. The reason seems to be a reduced capacity for digestion of phagocytosed material. A further exaggeration of these changes may possibly explain the glistening dots seen on ophthalmoscopy. However, more tissue rnaterial would be needed to ascertain whether this is true, or if retinal changes are involved as well.

We thank Dr Anders Eriksson, the State Institute of Forensic Medicine, Umeå, Sweden, for kindly providing the tissue material used in the present study, and Mrs Marianne Nordell for skilful technical assistance.

This investigation was supported by a grant from the Swedish Medical Research Council (Project No. 12X-734).

\section{References}

1 Sjögren T, Larsson T. Oligophrenia in combination with congenital ichthyosis and spastic disorders. Acta Psychiatr Scand 1957; 32(suppl 113): 1-113.

2 Gilbert WR Jr, Smith JL, Nyhan WL. The Sjögren-Larsson syndrome. Arch Ophthalmol 1968; 80: 308-16.

3 Theile U. Sjögren-Larsson syndrome. Oligophrenia-ichthyosisdi/tetraplegia. Hum Genet 1975; 22: 91-118.

4 Jagell S, Polland W, Sandgren O. Specific changes in the fundus typical for the Sjögren-Larsson syndrome. Acta Ophthalmol (Kbh) 1980; 58: 321-30.

5 Hernell O, Holmgren G, Jagell S, Jonson S, Holman RT. Suspected faulty essential fatty acid metabolism in SjögrenLarsson syndrome. Pediatr Res 1982; 16: 45-9.

6 Feeney-Burns L, Hilderbrand ES, Eldridge S. Aging human RPE: morphometric analysis of macular, equatorial, and peripheral cells. Invest Ophthalmol Vis Sci 1984; 25: 195-200.

Accepted for publication 9 May 1986. 